\title{
SOME RESULTS ON A GENERALIZATION OF ARMENDARIZ RINGS
}

\author{
ELTIYEB ALI ${ }^{1,2, *}$, AYOUB ELSHOKRY ${ }^{2}$ \\ ${ }^{1}$ Department of Mathematics, Faculty of Science and Arts, Najran University, sharourah, KSA \\ ${ }^{2}$ Department of Mathematics, Faculty of Education, University of Khartoum, Omdurman, Sudan \\ Corresponding author: eltiyeb76@gmail.com \\ Received Jan. 15, 2019
}

\begin{abstract}
Let $R$ be a ring, $(S, \leq)$ a strictly ordered monoid and $\omega: S \rightarrow \operatorname{End}(R)$ a monoid homomorphism. This paper is devoted to the study of $(S, \omega)$-nil-Armendariz under some additional conditions. Let $R$ be a completely $S$-compatible ring. We show that, if $I$ is semicommutative and $R / I$ is $(S, \bar{\omega})$-nil-Armendariz, then $R$ is $(S, \omega)$-nil-Armendariz. As a consequence, we deduce that for an $S$-compatible ring $R$, the skew generalized power series ring $\left[\left[R^{S, \leq}, \omega\right]\right]$ is weak symmetric (weakly reversible, weakly zip, nil-semicommutative) ring if and only if $R$ is weak symmetric (weakly reversible, weakly zip, nil-semicommutative, respectively). Moreover, some results of skew generalized power series $\left[\left[R^{S, \leq}, \omega\right]\right]$ are given.
\end{abstract}

2010 Mathematics Subject Classification. 16S99, 16W60, 16S36, 16 U80.

Key words and phrases. semicommutative ring; symmetric ring; zip ring; skew generalized power series ring; $(S, \omega)$ Armendariz ring.

\section{InTRODUCTION}

All rings considered here are associative with identity. We will write monoids multiplicatively unless otherwise indicated. If $R$ is a ring and $X$ is a nonempty subset of $R$, then the left (right) annihilator of $X$ in $R$ is denoted by $\ell_{R}(X)\left(r_{R}(X)\right)$. We will denote by $\operatorname{End}(R)$ the monoid of ring endomorphisms of $R$, and by $\operatorname{Aut}(R)$ the group of ring automorphisms of $R$.

Let $R$ be a ring, $(S, \leq)$ a strictly ordered monoid, and $\omega: S \rightarrow \operatorname{End}(R)$ a monoid homomorphism. For $s \in S$, let $\omega_{s}$ denote the image of $s$ under $\omega$, that is, $\omega_{s}=\omega(s)$. Let $A$ be the set of all functions $f: S \rightarrow R$ such that the $\operatorname{support} \operatorname{supp}(f)=\{s \in S: f(s) \neq 0\}$ is artinian and narrow. Then for any $s \in S$ and $f, g \in A$ the set

$$
X_{s}(f, g)=\{(u, v) \in \operatorname{supp}(f) \times \operatorname{supp}(g): s=u v\}
$$

is finite. Thus one can define the product $f g: S \rightarrow R$ of $f, g \in A$ as follows:

$$
(f g)(s)=\sum_{(u, v) \in X_{s}(f, g)} f(u) \omega_{u}(g(v))
$$

(by convention, a sum over the empty set is 0 ). With pointwise addition and multiplication as defined above, $A$ becomes a ring, called the ring of skew generalized power series with coefficients in $R$ and exponents in $S$, see [35] and denoted by $\left[\left[R^{S, \leq}, \omega\right]\right]$ (or by $R[[S, \omega]]$ when there is no ambiguity concerning the order $\leq$ ).

DOI: $10.28924 /$ APJM/6-1 
We will use the symbol 1 to denote the identity elements of the monoid $S$, the $\operatorname{ring} R$, and the ring $\left[\left[R^{S, \leq}, \omega\right]\right]$ as well as the trivial monoid homomorphism 1:S $\rightarrow \operatorname{End}(R)$ that sends every element of $S$ to the identity endomorphism. $A$ subset $P \subseteq R$ will be called $S$-invariant if for every $s \in S$ it is $\omega_{s}$-invariant (that is, $\omega_{s}(P) \subseteq P$ ). To each $r \in R$ and $s \in S$, we associate elements $c_{r}, e_{s} \in\left[\left[R^{S, \leq}, \omega\right]\right]$ defined by

$$
c_{r}(x)=\left\{\begin{array}{ll}
r, & x=1, \\
0, & x \in S \backslash\{1\},
\end{array} e_{s}(x)= \begin{cases}1, & x=s, \\
0, & x \in S \backslash\{s\} .\end{cases}\right.
$$

It is clear that $r \mapsto c_{r}$ is a ring embedding of $R$ into $\left[\left[R^{S, \leq}, \omega\right]\right]$ and $s \mapsto e_{s}$, is a monoid embedding of $S$ into the multiplicative monoid of the ring $\left[\left[R^{S, \leq}, \omega\right]\right]$, and $e_{s} c_{r}=c_{\omega_{s}(r)} e_{s}$.

Rege and Chhawchharia [25] introduced the notion of an Armendariz ring. They defined a ring $R$ to be an Armendariz ring if whenever polynomials $f(x)=a_{0}+a_{1} x+\cdots+a_{n} x^{n}, g(x)=b_{0}+b_{1} x+\cdots+b_{m} x^{m} \in R[x]$ satisfy $f(x) g(x)=0$, then $a_{i} b_{j}=0$ for each $i, j$. (The converse is always true.) The name "Armendariz ring" was chosen because Armendariz [13, Lemma 1] had noted that a reduced ring satisfies this condition. Reduced rings (i.e., rings with no nonzero nilpotent elements). Some properties of Armendariz rings have been studied in Rege and Chhawchharia [13], Anderson and Camillo [11], Kim and Lee [26], Huh, Lee and Smoktunowicz [18], and Lee and Wong [41].

Given a ring $R$ and a ring endomorphism $\sigma: R \rightarrow R$, the skew polynomial ring $R[x ; \sigma]$ consists of polynomials in the indeterminate $x$ with coefficients from $R$, written on the left, where multiplication in $R[x ; \sigma]$ is defined by

$$
\left(\sum_{i} a_{i} x^{i}\right)\left(\sum_{j} b_{j} x^{j}\right)=\sum_{i, j} a_{i} \sigma^{i}\left(b_{j}\right) x^{i+j} .
$$

Following Hong et al. [9], we say that a ring $R$ with an endomorphism $\sigma$ is $\sigma$-skew Armendariz if whenever polynomials $f(x)=a_{0}+a_{1} x+\cdots+a_{n} x^{n}$ and $g(x)=b_{0}+b_{1} x+\cdots+b_{m} x^{m}$ in $R[x ; \sigma]$ satisfy $f(x) g(x)=0$ then $a_{i} \sigma^{i}\left(b_{j}\right)=0$ for all $i, j$. A stronger condition than Armendariz was studied by Kim et al. in [27]. A ring $R$ is said to be power-serieswise Armendariz if whenever power series $f(x)=a_{0}+a_{1} x+\cdots+a_{n} x^{n}$ and $g(x)=b_{0}+b_{1} x+\cdots+b_{m} x^{m}$ in $R[[x]]$ satisfy $f(x) g(x)=0$ then $a_{i} b_{j}=0$ for all $i, j$.

If $R$ is a ring and $S$ is a strictly ordered monoid, then the ring $R$ is called a generalized Armendariz ring if for each $f, g \in\left[\left[R^{S, \leq}\right]\right]$ such that $f g=0$ implies that $f\left(u_{i}\right) g\left(v_{j}\right)=0$ for each $u_{i} \in \operatorname{supp}(f)$ and $v_{j} \in \operatorname{supp}(g)$. In [45] Liu called such ring $S$-Armendariz ring. He proved by [45, Lemma 3.1] a reduced rings are $S$-Armendariz for any torsion free and cancellative monoid $S$.

This paper is devoted to continue to study some results of $(S, \omega)$-nil-Armendariz ring under some additional conditions, which is a common generalization of $S$-Armendariz, $(S, \omega)$-Armendariz, nil power serieswise Armendariz rings and nil generalized power serieswise Armendariz rings. For a ring $R,(S, \leq)$ a strictly ordered monoid and $\omega: S \rightarrow \operatorname{End}(R)$ a monoid homomorphism and $R$ be a completely $S$-compatible ring. We show that, if $I$ is semicommutative and $R / I$ is $(S, \bar{\omega})$-nil-Armendariz, then $R$ is $(S, \omega)$-nil-Armendariz. As a consequence, we deduce that for an $S$-compatible ring $R$, the skew generalized power series ring $\left[\left[R^{S, \leq}, \omega\right]\right]$ is weak symmetric (weakly reversible, weakly zip, nil-semicommutative) ring if and only if $R$ is weak symmetric (weakly reversible, 
weakly zip, nil-semicommutative, respectively). Moreover, several known results relating to skew generalized power series $\left[\left[R^{S, \leq}, \omega\right]\right]$ can be obtained as corollaries of our results.

\section{2. (S, $\omega)$-nil-Armendariz rings}

The Wedderburn radical (i.e., the largest nilpotent ideal in $R$ ), the lower nil radical (i.e., the intersection of all the prime ideals in $R$ ), the Levitzky radical (i.e., sum of all locally nilpotent ideals), the upper nil radical (i.e., sum of all nil ideals), the set of all nilpotent elements of $R$ and the sum of all nil left ideals of $R$ (which coincides with the sum of all nil right ideals of $R$ ) is denoted by $N_{0}(R), N i l_{*}(R), L-\operatorname{rad}(R), N i l^{*}(R), N i l(R)$ and $A(R)$, respectively.

One can find the next definitions in [16, Definition 2.1] and our P.hD [14] and [23] respectively.

Definition 2.1. Let $R$ be a ring, $(S, \leq)$ be a strictly ordered monoid, and $\omega: S \rightarrow E n d(R)$ a monoid homomorphism. The ring $R$ is called $(S, \omega)$-Armendariz, if whenever $f g=0$ for $f, g \in\left[\left[R^{S, \leq}, \omega\right]\right]$, then $f(u) \omega_{u}(g(v))=0$ for each $u, v \in S$.

Definition 2.2. Let $R$ be a ring, $(S, \leq)$ be a strictly ordered monoid, and $\omega: S \rightarrow E n d(R)$ a monoid homomorphism. We say that $R$ is $(S, \omega)$-nil-Armendariz, if whenever $f, g \in\left[\left[R^{S, \leq}, \omega\right]\right]$ satisfy $f g \in\left[\left[\operatorname{nil}(R)^{S, \leq}, \omega\right]\right]$, then $f(u) \omega_{u}(g(v)) \in \operatorname{nil}(R)$ for each $u, v \in S$.

Let $S=(\mathbb{N} \cup\{0\},+)$ and $\leq$ is the usual order. Then, $\left[\left[R^{S, \leq}, \omega\right]\right] \cong R[[x]]$. Let $\omega$ be the trivial order. Then the ring $R$ is $(S, \omega)$-nil-Armendariz, if and only if, $R$ is nil power series Armendariz. Hence $(S, \omega)$-nil-Armendariz ring is a common generalization of above. If $T$ is a subring of $R$ and $\omega_{u}(T) \subseteq T$ for any $u \in S$, then $T$ is $(S, \omega)$-nil-Armendariz, when $R$ is $(S, \omega)$-nil-Armendariz. A ring $R$ is called an $N I$ ring if $n i l(R)$ forms an ideal.

According to Krempa [19], an endomorphism $\alpha$ of a ring $R$ is said to be rigid if $a \alpha(a)=0$ implies $a=0$ for $a \in R$. A ring $R$ is said to be $\alpha$-rigid if there exists a rigid endomorphism $\alpha$ of $R$. And $\alpha$-rigid rings are reduced rings by [10, Proposition 5]. In [12], the authors introduced $\alpha$-compatible rings and studied their properties. A $\operatorname{ring} R$ is $\alpha$-compatible if for each $a, b \in R, a b=0$ if and only if $a \alpha(b)=0$.

Basic properties of rigid and compatible endomorphisms, proved by Hashemi and Moussavi are summarized in what follows:

Lemma 2.3. Let $\alpha$ be an endomorphism of a ring $R$.

(1) If $\alpha$ is compatible, then $\alpha$ is injective.

(2) $\alpha$ is compatible if and only if for all $a, b \in R, \alpha(a) b=0 \Leftrightarrow a b=0$.

(3) The following conditions are equivalent:

(a) $\alpha$ is rigid;

(b) $\alpha$ is compatible and $R$ is reduced;

(c) For every $a \in R, \alpha(a) a=0$ implies that $a=0$.

Definition 2.4. [16] Let $R$ be a ring, $(S, \leq)$ a strictly ordered monoid and $\omega: S \rightarrow E n d(R)$ a monoid homomorphism. The ring $R$ is said to be $S$-compatible (resp. $S$-rigid) if $\omega_{s}$ is compatible (resp. rigid) for every $s \in S$; to indicate the homomorphism $\omega$, we will sometimes say that $R$ is $(S, \omega)$-compatible (resp. $(S, \omega)$-rigid). 
Lemma 2.5. Let $\omega: S \rightarrow \operatorname{End}(R)$ a monoid homomorphism. For each $a, b \in R$, each $s \in S$, the followings holds:

(1) $a b \in \operatorname{nil}(R) \Leftrightarrow a \omega_{s}(b) \in \operatorname{nil}(R)$.

(2) $a b \in \operatorname{nil}(R) \Leftrightarrow \omega_{s}(a) b \in \operatorname{nil}(R)$.

Proof. It is enough to proof (1) and the proof of (2) is similar. Suppose that $a b \in \operatorname{nil}(R)$ for each $a, b \in R$. Then there exists some positive integer $k$ such that $(a b)^{k}=0$. Using the condition that $\omega$ is compatible, we have

$0=(a b)^{k}=a b a b \cdots a b$

$\Leftrightarrow a b a b \cdots a b a b a \omega_{s}(b)=0$

$\Leftrightarrow a b a b \cdots a \omega_{s}\left((b) a \omega_{s}(b)\right)=0$

$\Leftrightarrow a b a b \cdots a \omega_{s}(b) \omega_{s}\left(a \omega_{s}(b)\right)=0$

$\Leftrightarrow a b a b a b \cdots a \omega_{s}(b) a \omega_{s}(b)=0$

$\cdots a \omega_{s}(b) a \omega_{s}(b) \cdots a \omega_{s}(b) a \omega_{s}(b)=0$

Therefore $a w_{s}(b) \in \operatorname{nil}(R)$.

The following result appeared in [16].

Lemma 2.6. Let $R$ be a ring, $(S, \leq)$ a strictly ordered monoid and $\omega: S \rightarrow \operatorname{End}(R)$ a monoid homomorphism. Then $\left[\left[R^{S, \leq}, \omega\right]\right]$ is reduced if and only if $R$ is reduced.

Let $R$ be a ring, $\left(S_{1}, \leq_{1}\right),\left(S_{2}, \leq_{2}\right), \ldots,\left(S_{n}, \leq_{n}\right)$ be strictly ordered monoid, and $\omega^{i}: S_{i} \rightarrow \operatorname{End}(R)$ be a monoid homomorphism for every $i$. Define $\omega: S \rightarrow \operatorname{End}(R)$ as

$$
\omega\left(s_{1}, s_{2}, \ldots, s_{n}\right)=\omega_{s_{1}} \omega_{s_{2}} \cdots \omega_{s_{n}}
$$

That is,

$$
\omega_{\left(s_{1}, s_{2}, \ldots, s_{n}\right)}=\omega_{s_{1}} \omega_{s_{2}} \cdots \omega_{s_{n}} .
$$

Then $\omega$ is well-defined.

Lemma 2.7. If $R$ is $S_{i}$-compatible for each $i$, then $R$ is $S$-compatible.

Proof. Let $a, b \in R$. Then for any $s_{i} \in S$,

$a b=0 \Leftrightarrow a \omega_{s_{n}}(b)=0$

$\Leftrightarrow a \omega_{s_{n-1}} \omega_{s_{n}}(b)=0$

$\cdots$

$\Leftrightarrow a \omega_{s_{1}} \omega_{s_{2}} \cdots \omega_{s_{n}}(b)=0$

$\Leftrightarrow a \omega_{\left(s_{1}, s_{2}, \ldots, s_{n}\right)}(b)=0$.

Thus, $R$ is $S$-compatible.

Proposition 2.8. Let $R$ be a ring, $(S, \leq)$ a strictly ordered monoid, and $\omega: S \rightarrow \operatorname{End}(R)$ a monoid homomorphism such that $R$ is $S$-compatible. Then the following conditions are equivalent:

(1) $R$ is $(S, \omega)$-nil-Armendariz.

(2) $R$ is an NI ring. 
Proof. (1) $\Rightarrow(2)$ Since $R$ is $S$-compatible the proof is similar to the proof of [29, Proposition 2.2(1)].

$(2) \Rightarrow(1)$ Assume that $R$ is an $N I$ ring and $f, g \in\left[\left[R^{S, \leq}, \omega\right]\right]$ are such that $f g \in\left[\left[\operatorname{nil}(R)^{S, \leq}, \omega\right]\right]$. Then $\bar{f} \bar{g}=\overline{0}$, where $\bar{f}, \bar{g}$ are the corresponding skew generalized power series of $f, g$ in $\left[\left[(R / n i l(R))^{S, \leq}, \omega\right]\right]$. Observe that $R / \operatorname{nil}(R)$ is reduced and hence $(S, \omega)$-Armendariz by [16]. Thus, $\bar{f}(u) \bar{\omega}_{u}(\bar{g}(v))=\overline{0}$, where $\bar{\omega}_{u}(\bar{g}(v))=$ $\omega_{u}(g(v)+n i l(R))$. Note that the $S$-compatibility implies $\bar{\omega}_{u}$ is well-defined. So, $\bar{f}(u) \bar{g}(v)=\overline{0}$ for all $u, v \in S$. Hence $f(u) g(v) \in \operatorname{nil}(R)$ for any $u, v \in S$. Therefore $R$ is $(S, \omega)$-nil-Armendariz by Lemma 2.5.

Corollary 2.9. Let $R$ be a ring, $\left(S_{1}, \leq_{1}\right),\left(S_{2}, \leq_{2}\right), \ldots,\left(S_{n}, \leq_{n}\right)$ be a strictly ordered monoids, and $\omega$ is defined as above. Denote by $($ lex $\leq)$ and (revlex $\leq)$ the lexicographic order, the reverse lexicographic order, respectively, on the monoid $S_{1} \times S_{2} \times \cdots \times S_{n}$. Then the following conditions are equivalent:

(1) $R$ is an NI ring.

(2) $R$ is $\left(S_{1} \times S_{2} \times \cdots \times S_{n}, \omega\right)$-nil-Armendariz for any ordered monoid $\left(S_{i}, \leq_{i}\right)$.

(3) $R$ is $\left(S_{1} \times S_{2} \times \cdots \times S_{n}, \omega\right)$-nil-Armendariz for ordered monoid $\left(S_{1} \times S_{2} \times \cdots \times S_{n},(\right.$ lex $\left.\leq)\right)$.

(4) $R$ is $\left(S_{1} \times S_{2} \times \cdots \times S_{n}, \omega\right)$-nil-Armendariz for ordered monoid $\left(S_{1} \times S_{2} \times \cdots \times S_{n},(\right.$ revlex $\left.\leq)\right)$.

Proof. It is easy to see that $\left(S_{1} \times S_{2} \times \cdots \times S_{n},(\right.$ lex $\left.\leq)\right)$ and $\left(S_{1} \times S_{2} \times \cdots \times S_{n},(\right.$ revlex $\left.\leq)\right)$ are strictly ordered monoids. Therefore we complete the proofs of $(2) \Leftrightarrow(1),(2) \Leftrightarrow(3)$ and $(2) \Leftrightarrow(4)$ by Proposition 2.8 .

In [29]. A ring $R$ is called $n$ nil power serieswise Armendariz if $f=\Sigma a_{i_{1}, i_{2}, \ldots, i_{n}} x_{1}^{i_{1}} x_{2}^{i_{2}}$ $\cdots x_{n}^{i_{n}}, g=\Sigma b_{j_{1}, j_{2}, \ldots, j_{m}} x_{1}^{j_{1}} x_{2}^{j_{2}} \cdots x_{n}^{j_{n}} \in R\left[\left[x_{1}, x_{2}, \ldots, x_{n}\right]\right]$ satisfy $f g \in \operatorname{nil}(R)\left[\left[x_{1}, x_{2}, \cdots\right.\right.$ , $\left.\left.x_{n}\right]\right]$, then $a_{i_{1}, i_{2}, \ldots, i_{n}} b_{j_{1}, j_{2}, \ldots, j_{n}} \in \operatorname{nil}(R)$ for all for all $i_{1}, i_{2}, \ldots, i_{n}$ and $j_{1}, j_{2}, \ldots, j_{n}$.

Corollary 2.10. [29, Corollary 2.4] Let $R$ be a ring. Then the following conditions are equivalent.

(1) The ring $R$ is an NI ring.

(2) The ring $R$ is nil power series Armendariz.

(3) The ring $R$ is $n$ nil power series Armendariz.

Lemma 2.11. Let $R$ be a ring, $(S, \leq)$ a strictly ordered monoid and $\omega: S \rightarrow E n d(R)$ a monoid homomorphism. If $R$ is $S$-compatible $(S, \omega)$-Armendariz, then for any $f_{1}, f_{2}, \ldots, f_{n}$ $\in\left[\left[R^{S, \leq}, \omega\right]\right]$ with $f_{1} f_{2} \cdots f_{n}=0, f_{1}\left(u_{1}\right) f_{2}\left(u_{2}\right) \cdots f_{n}\left(u_{n}\right)=0$ for any $u_{1}, u_{2}, \ldots, u_{n} \in S$.

Proof. Using Lemma 2.5, the proof is similar to that of [45, Proposition 3.2].

Corollary 2.12. Let $R$ be a ring, $(S, \leq)$ a ordered commutative torsion-free and cancellative monoid. Assume that $R$ is $S$-rigid and $\omega: S \rightarrow \operatorname{End}(R)$ a monoid homomorphism. Then for each $f_{1}, f_{2}, \ldots, f_{n} \in\left[\left[R^{S, \leq}, \omega\right]\right], f_{1} f_{2} \cdots f_{n}=0$, implies $f_{1}\left(u_{1}\right) f_{2}\left(u_{2}\right) \cdots f_{n}\left(u_{n}\right)$ $=0$ for any $u_{1}, u_{2}, \ldots, u_{n} \in S$.

Proof. Immediately from [16, Theorem 4.12] and Lemma 2.11.

Proposition 2.13. Let $R$ be a ring, $(S, \leq)$ a strictly ordered monoid and $w: S \rightarrow \operatorname{End}(R)$ a monoid homomorphism. If $R$ is $S$-compatible and $(S, \omega)$-nil-Armendariz, then for any $f_{1}, \ldots, f_{n} \in\left[\left[R^{S, \leq}, \omega\right]\right]$ with $f_{1} f_{2} \cdots f_{n} \in\left[\left[n i l(R)^{S, \leq}, \omega\right]\right]$, $f_{1}\left(u_{1}\right) f_{2}\left(u_{2}\right) \cdots$ $f_{n}\left(u_{n}\right) \in \operatorname{nil}(R)$ for any $u_{1}, u_{2}, \ldots, u_{n} \in S$. 
Proof. Using Lemma 2.5 freely. The proof is by induction on $n$. The case $n=2$ is clear by the definition of $(S, \omega)$-nil-Armendariz ring. Suppose that $f_{1}, f_{2}, \ldots, f_{n} \in\left[\left[R^{S, \leq}, \omega\right]\right]$ are such that $f_{1} f_{2} \cdots f_{n} \in\left[\left[n i l(R)^{S, \leq}, \omega\right]\right]$ for $n>2$. Then from $f_{1}\left(f_{2} f_{3} \cdots f_{n}\right)$

$\in\left[\left[\operatorname{nil}(R)^{S, \leq}, \omega\right]\right]$ it follows that $f_{1}\left(u_{1}\right)\left(f_{2} f_{3} \cdots f_{n}\right)(v) \in \operatorname{nil}(R)$ for any $u_{1}, v \in S$ since $R$ is $S$-compatible.

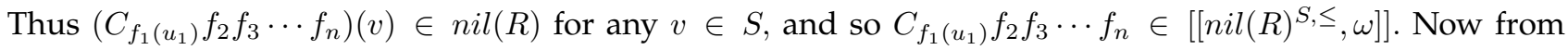
$\left(C_{f_{1}\left(u_{1}\right)} f_{2}\right)\left(f_{3} \cdots f_{n}\right) \in\left[\left[\operatorname{nil}(R)^{S, \leq}, \omega\right]\right]$, it follows that $\left(C_{f_{1}\left(u_{1}\right)} f_{2}\right)\left(u_{2}\right)$ $\left(f_{3} \cdots f_{n}\right)(z) \in \operatorname{nil}(R)$ for all $u_{2}, z \in S$. Since

$$
\left(C_{f_{1}\left(u_{1}\right)} f_{2}\right)\left(u_{2}\right)=f_{1}\left(u_{1}\right) f_{2}\left(u_{2}\right)
$$

for any $u_{1}, u_{2} \in S$, we have

$$
f_{1}\left(u_{1}\right) f_{2}\left(u_{2}\right)\left(f_{3} f_{4} \cdots f_{n}\right)(z) \in \operatorname{nil}(R)
$$

for all $u_{1}, u_{2}, z \in S$. Hence

$$
C_{\left(f_{1}\left(u_{1}\right) f_{2}\left(u_{2}\right)\right)} f_{3} f_{4} \cdots f_{n} \in\left[\left[\operatorname{nil}(R)^{S, \leq}, \omega\right]\right] .
$$

Continuing this manner, we see that $\left.f_{1}\left(u_{1}\right) f_{2}\right)\left(u_{2}\right) \cdots f_{n}\left(u_{n}\right) \in \operatorname{nil}(R)$ for all $u_{1}, u_{2}$, $\ldots, u_{n} \in S$.

Corollary 2.14. Let $R$ be a ring, $(S, \leq)$ a strictly ordered monoid and $w: S \rightarrow E n d(R)$ a monoid homomorphism and $R$ is $S$-compatible. Then the following conditions are equivalents:

(1) If $f_{1}, f_{2}, \ldots, f_{n} \in\left[\left[R^{S, \leq}, \omega\right]\right]$ satisfy $f_{1} f_{2} \cdots f_{n} \in\left[\left[\operatorname{nil}(R)^{S, \leq}, \omega\right]\right]$, then $f_{1}\left(u_{1}\right)$ $f_{2}\left(u_{2}\right) \cdots f_{n}\left(u_{n}\right) \in \operatorname{nil}(R)$, for any $u_{1}, u_{2}, \ldots, u_{n} \in S$.

(2) $R$ is NI ring.

Proposition 2.15. Let $R$ be a ring, $(S, \leq)$ a strictly ordered monoid and $\omega: S \rightarrow \operatorname{End}(R)$ a compatible monoid homomorphism such that $\operatorname{nil}(R) \unlhd R$. Then if $f g \in\left[\left[\operatorname{nil}(R)^{S, \leq}, \omega\right]\right], f(u) g(v) \in \operatorname{nil}(R)$ for all $u, v \in S$.

Proof. Sinse $\operatorname{nil}(R)$ is an ideal of $R$, then $R$ is $N I$ ring. Thus the proof is similar to the proof of Proposition 2.8 .

Hashemi and Esmaili [19], shows that there exists a ring $R$ and an endomorphism $\alpha$ on $R$ such that $\operatorname{nil}(R)$ is an $\alpha$-compatible ideal of $R$. Here we have corollary.

Corollary 2.16. Let $R$ be a ring such that nil $(R)$ is an $S$-compatible ideal of $R,(S, \leq)$ a strictly ordered monoid and $\omega: S \rightarrow \operatorname{End}(R)$ monoid homomorphism. Then if $f g \in\left[\left[\operatorname{nil}(R)^{S, \leq}, \omega\right]\right], f(u) g(v) \in \operatorname{nil}(R)$ for each $u, v \in S$.

Observe that if $\operatorname{nil}(R) \unlhd R$, then by Proposition 2.15, $R$ is $(S, \omega)$-nil-Armendariz. More generally we obtain the following.

Lemma 2.17. Let $R$ be a ring, $(S, \leq)$ a strictly ordered monoid, and $\omega: S \rightarrow E n d(R)$ a monoid homomorphism. Assume that $R$ is $S$-compatible and $(S, \omega)$-Armendariz, then $\operatorname{nil}\left(\left[\left[R^{S, \leq}, \omega\right]\right]\right) \subseteq\left[\left[\operatorname{nil}(R)^{S, \leq}, \omega\right]\right]$.

Proof. Let $f \in \operatorname{nil}\left(\left[\left[R^{S, \leq}, \omega\right]\right]\right)$ and assume that $f^{k}=0$ where $k \in \mathbb{Z}$. Then by Proposition 2.13, $f(u) \in \operatorname{nil}(R)$ for any $u \in S$. Hence $\operatorname{nil}\left(\left[\left[R^{S, \leq}, \omega\right]\right]\right) \subseteq\left[\left[\operatorname{nil}(R)^{S, \leq}, \omega\right]\right]$. 
In [16], a ring $R$ is called linearly $(S, \omega)$-Armendariz if for all $s \in S \backslash\{1\}$ and $a_{0}, a_{1}, b_{0}, b_{1}$

$\in R$, whenever $\left(c_{a_{0}}+c_{a_{1}} e_{s}\right)\left(c_{b_{0}}+c_{b_{1}} e_{s}\right)=0$ in $\left[\left[R^{S, \leq}, \omega\right]\right]$, then $a_{0} b_{0}=a_{0} b_{1}=a_{1} \omega_{s}\left(b_{0}\right)=a_{1} \omega_{s}\left(b_{1}\right)=0$ in $R$.

The following result appeared in [2].

Corollary 2.18. Let $R$ be a ring, $(S, \leq)$ a strictly ordered monoid and $\omega: S \rightarrow E n d(R)$ a monoid homomorphism. If $R$ is linearly $(S, \omega)$-Armendariz and $\omega_{s}$ is compatible for some $s \in S \backslash\{1\}$, then:

(1) $\operatorname{Nil}(R)$ is a (nonunital) subring of $R$;

(2) $N_{0}(R)=N i l_{*}(R)=L-\operatorname{rad}(R)=N i l^{*}(R)=A(R)$. In particular, the Köthe problem has a positive solution in the class of $S$-compatible linearly $(S, \omega)$-Armendariz rings.

Concerning the question of whether $\operatorname{nil}(R[x])=\operatorname{nil}(R)[x]$, Amitsur [4], proved that this is true for $K$-algebras over uncountable fields. But recently, Smoktunowicz, in [1], has proved that the result is not true for algebras over countable fields.

Proposition 2.19. Let $R$ be NI ring, $(S, \leq)$ a strictly ordered monoid, and $\omega: S \rightarrow \operatorname{End}(R)$ a monoid homomorphism. If $R$ is $S$-compatible $(S, \omega)$-nil-Armendariz and $n i l(R)$ is an ideal of $R$, then

$$
\left[\left[\operatorname{nil}(R)^{S, \leq}, \omega\right]\right]=\operatorname{nil}\left(\left[\left[R^{S, \leq}, \omega\right]\right]\right) .
$$

Proof. Let $a \in \operatorname{nil}(R)$, then by Corollary 2.18, $R a R$ is a nilpotent in $R$. Since $R$ is $S$-compatible, for each $s \in S$, $R \omega_{s}(a) R$ is a nilpotent ideal of $R$ and so $\omega_{s}(a) \in \operatorname{nil}(R)$. Thus $\operatorname{nil}(R)$ is $S$-invariant and so $\left[\left[n i l(R)^{S, \leq}, \omega\right]\right]$ is an ideal of $\left[\left[R^{S, \leq}, \omega\right]\right]$. By Lemma 2.17, we have $\operatorname{nil}\left(\left[\left[R^{S, \leq}, \omega\right]\right]\right) \subseteq\left[\left[\operatorname{nil}(R)^{S, \leq}, \omega\right]\right]$. So it is suffices to show that $\left[\left[\operatorname{nil}(R)^{S, \leq}, \omega\right]\right] \subseteq \operatorname{nil}\left(\left[\left[R^{S, \leq}, \omega\right]\right]\right)$.

Assume that $f \in\left[\left[\operatorname{nil}(R)^{S, \leq}, \omega\right]\right]$ then for any $u \in S, f(u) \in \operatorname{nil}(R)$. By Corollary 2.18 there exists some positive integer $k$ such that for each $u \in S,(R f(u) R)^{k}=0$. Since $R$ is $S$-compatible, then for each $g, h \in$ $\left[\left[R^{S, \leq}, \omega\right]\right],(g f h)^{k}=0$. By Lemma 2.17, we know that if $g \in \operatorname{nil}\left(\left[\left[R^{S, \leq}, \omega\right]\right]\right)$, then $f(u) \in \operatorname{nil}(R)$, for each $u \in S$. So $f \in \operatorname{nil}\left(\left[\left[R^{S, \leq}, \omega\right]\right]\right)$. Hence $\left[\left[\operatorname{nil}(R)^{S, \leq}, \omega\right]\right] \subseteq \operatorname{nil}\left(\left[\left[R^{S, \leq}, \omega\right]\right]\right)$. Thus, $\left[\left[\operatorname{nil}(R)^{S, \leq}, \omega\right]\right]=\operatorname{nil}\left(\left[\left[R^{S, \leq}, \omega\right]\right]\right)$.

Corollary 2.20. Let $R$ be a ring, $(S, \leq)$ a strictly ordered monoid, and $\omega: S \rightarrow \operatorname{End}(R)$ a monoid homomorphism. If $R$ is $S$-compatible and $(S, \omega)$-nil-Armendariz right noetherian ring, then $\left[\left[\operatorname{nil}(R)^{S, \leq}, \omega\right]\right]=\operatorname{nil}\left(\left[\left[R^{S, \leq}, \omega\right]\right]\right)$.

Proof. Since $R$ is $(S, \omega)$-nil-Armendariz, by Proposition 2.8, $R$ is an $N I$ ring, $n i l(R)$ is nilpotent. Hence the result follows from Proposition 2.19 .

From Lemma 2.17 and Proposition 2.19, we have the following corollary.

Corollary 2.21. Let $R$ be a ring, $(S, \leq)$ a strictly ordered monoid, $\omega: S \rightarrow \operatorname{End}(R)$ a monoid homomorphism and $R$ is $S$-compatible $(S, \omega)$-Armendariz. Then $f$ is a nilpotent element of $\left[\left[R^{S, \leq}, \omega\right]\right]$ if and only if $f(u) \in \operatorname{nil}(R)$ for each $u \in S$.

A ring $R$ is called semicommutative if for all $a, b \in R, a b=0$ implies $a R b=0$. This is equivalent to the usual definition by Shin [40, Lemma 1.2] or Huh et al. [18, Lemma 1]. By Huh et al. [18], reduced rings are semicommutative, and semicommutative rings are Abelian (i.e., rings in which every idempotent is central). N. K. Kim et al. have shown in [27, Proposition 3.1] that a ring $R$ is power serieswise Armendariz if and only if $R[x]$ is power serieswise Armendariz. For nil power serieswise Armendariz rings, S. Hizem have shown in [38, Corollary 
7] that if $R$ is a semicommutative ring, then $R[x]$ is a nil power serieswise Armendariz ring. Ouyang and Liu [29] it was shown that if $\operatorname{nil}(R)$ is nilpotent and $R$ is nil generalized power serieswise Armendariz rings, then $\left[\left[R^{S, \leq}\right]\right]$ is nil generalized power serieswise Armendariz rings. As to $(S, \omega)$-nil-Armendariz rings, we have the following.

Proposition 2.22. Let $R$ be a ring, $(S, \leq)$ a strictly ordered monoid and $\omega: S \rightarrow \operatorname{End}(R)$ a monoid homomorphism and $R$ is $(S, \omega)$-nil-Armendariz. If $R$ is $S$-compatible and $n i l(R)$ is nilpotent, then $\left[\left[R^{S, \leq}, \omega\right]\right]$ is nil-Armendariz for any strictly ordered monoid $\left(T, \leq_{T}\right)$.

Proof. Since $R$ is $(S, \omega)$-nil-Armendariz, by Proposition 2.8, $R$ is an $N I$ ring. Since $n i l(R)$ is nilpotent, by Proposition 2.19, we have $\left[\left[\operatorname{nil}(R)^{S, \leq}, \omega\right]\right]=\operatorname{nil}\left(\left[\left[R^{S, \leq}, \omega\right]\right]\right)$, and so $\left[\left[R^{S, \leq}, \omega\right]\right]$ is an $N I$ ring. Then by Proposition 2.8, $\left[\left[R^{S, \leq}, \omega\right]\right]$ is nil-Armendariz for any strictly ordered monoid $\left(T, \leq_{T}\right)$.

Corollary 2.23. Let $(S, \leq)$ a strictly ordered monoid and $\omega: S \rightarrow \operatorname{End}(R)$ a monoid homomorphism and $R$ is $(S, \omega)$-nilArmendariz right noetherian ring. If $R$ is $S$-compatible, then $\left[\left[R^{S, \leq}, \omega\right]\right]$ is nil-Armendariz for any strictly ordered monoid $\left(T, \leq_{T}\right)$.

Following [39], we say that a module $M_{R}$ is completely $\sigma$-compatible if, for every $N_{R} \subseteq M_{R}$, we have that $(M / N)_{R}$ is $\sigma$-compatible. Here we have.

Definition 2.24. Let $R$ be a ring, $(S, \leq)$ a strictly ordered monoid and $\omega: S \rightarrow E n d(R)$ a monoid homomorphism. We say that a ring $R$ is completely $S$-compatible if, for any ideal $I$ of $R, R / I$ is $S$-compatible, to indicate the homomorphism $\omega$, we will sometimes say that $R$ is completely $(S, \omega)$-compatible.

Clearly, every completely $S$-compatible ring is $S$-compatible. Another description of complete $S$-compatibility of $R$ that we shall often use is that for all $I \subseteq R, a, b \in R$, we have $a b \in I \Leftrightarrow a \omega(b) \in I$.

Qun and Wang in [29, Proposition 2.12] it was shown that, if $I$ is semicommutative such that $R / I$ is nil generalized power series Armendariz, then $R$ is nil generalized power series Armendariz. Here we have the following result.

Theorem 2.25. Let $R$ be a ring, $(S, \leq)$ a strictly ordered monoid, $\omega: S \rightarrow \operatorname{End}(R)$ a monoid homomorphism and $I$ an ideal of $R$. Assume that $R$ is a completely $S$-compatible ring. If $I$ is semicommutative and $R / I$ is $(S, \bar{\omega})$-nil-Armendariz, where $\bar{\omega}: S \rightarrow \operatorname{End}(R / I)$ is the induced monoid homomorphism, then $R$ is $(S, \omega)$-nil-Armendariz.

Proof. Let $f, g \in\left[\left[R^{S, \leq}, \omega\right]\right]$ be such that $f g \in\left[\left[\operatorname{nil}(R)^{S, \leq}, \omega\right]\right]$. By Ribenbiom [32], there exists a compatible strict total order $\leq^{\prime}$ on $S$, which is finer than $\leq$ (that is, for all $u_{0}, v_{0} \in S, u_{0} \leq v_{0}$ implies $u_{0} \leq^{\prime} v_{0}$ ). We will use transfinite induction on the strictly totally ordered set $\left(S, \leq^{\prime}\right)$ to show that $f(u) g(v) \in \operatorname{nil}(R)$ for any $u, v \in S$. Let $u_{0}$ and $v_{0}$ denote the minimum elements of $\operatorname{supp}(f)$ and $\operatorname{supp}(g)$ in the $\leq^{\prime}$ order, respectively. If $u \in \operatorname{supp}(f)$ and $v \in \operatorname{supp}(g)$ are such that $u+v=u_{0}+v_{0}$, then $u_{0} \leq^{\prime} u$ and $v_{0} \leq^{\prime} v$. If $u_{0}<^{\prime} u$, then $u_{0}+v_{0}<^{\prime} u+v=u_{0}+v_{0}$, a contradiction. Thus $u=u_{0}$. Similarly, $v=v_{0}$. Hence

$$
(f g)\left(u_{0}+v_{0}\right)=\sum_{(u, v) \in X_{\left(u_{0}, v_{0}\right)}(f, g)} f(u) \omega_{u}(g(v))=f\left(u_{0}\right) \omega_{u_{0}}\left(g\left(v_{0}\right)\right) \in \operatorname{nil}(R)
$$

because $f g \in\left[\left[\operatorname{nil}(R)^{S, \leq}, \omega\right]\right]$. 
Now, suppose that $s \in S$ is such that for any $u, v \in S$ with $u+v<^{\prime} s, f(u) g(v) \in \operatorname{nil}(R)$. We will show that $f(u) g(v) \in \operatorname{nil}(R)$ for any $u, v \in S$ with $u+v=s$. We write

$$
X_{s}(f, g)=\{(u, v) \in S \times S \mid u+v=s, u \in \operatorname{supp}(f), v \in \operatorname{supp}(g)\}
$$

as $\left\{\left(u_{i}, v_{i}\right) \mid i=1, \ldots, n\right\}$ such that

$$
u_{1}<^{\prime} u_{2}<^{\prime} \cdots<^{\prime} u_{n}
$$

Since $S$ is cancellative, $u_{1}=u_{2}$ and $u_{1}+v_{1}=u_{2}+v_{2}=s$, implies $v_{1}=v_{2}$. Since $<^{\prime}$ is a strictly order, $u_{1}<^{\prime} u_{2}$ and $u_{1}+v_{1}=u_{2}+v_{2}=s$ implies $v_{2}<^{\prime} v_{1}$. Thus we have

$$
v_{n}<^{\prime} v_{n-1}<^{\prime} \cdots<^{\prime} v_{2}<^{\prime} v_{1} .
$$

Now,

$$
(f g)(s)=\sum_{(u, v) \in X_{s}(f, g)} f(u) \omega_{u}(g(v))=\sum_{i=1}^{n} f\left(u_{i}\right) \omega_{u_{i}}\left(g\left(v_{i}\right)\right) \in \operatorname{nil}(R) .
$$

For any $i \geq 2, u_{1}+v_{i}<^{\prime} u_{i}+v_{i}=s$, and thus, by induction hypothesis, we have $f\left(u_{1}\right) g\left(v_{i}\right) \in \operatorname{nil}(R)$.

On the other hand, if we denote by $\bar{f}, \bar{g}$ the corresponding skew generalized power series of $f$ and $g$ in $\left[\left[(R / I)^{S, \leq}, \omega\right]\right], \bar{f} \bar{g} \in\left[\left[(\operatorname{nil}(R / I))^{S, \leq}, \omega\right]\right]$. Using the definition 2.24 and Lemma 2.5, there exists $n_{i j} \in \mathbb{N}$ such that $\left(f\left(u_{i}\right) g\left(v_{j}\right)\right)^{n_{i j}} \in I$ since $R / I$ is $(S, \bar{\omega})$-nil-Armendariz. Then by analogy with the proof of Liu and Zhao [44], Theorem 3.6, we can show that $f(u) g(v) \in \operatorname{nil}(R)$ for any $u, v \in S$ with $u+v=s$. Hence by transfinite induction, $f(u) g(v) \in \operatorname{nil}(R)$ for any $u, v \in S$. Therefore $R$ is $(S, \omega)$-nil-Armendariz.

Definition 2.26. [22] Let $R$ be a ring. We say that $R$ has nil-semicommutative, if $a b=0$ implies arb $=0$, for each $r \in \operatorname{nil}(R)$ and $\operatorname{arb} \in R$, where $\operatorname{nil}(R)$ is the set of nilpotent elements of $R$.

Clearly, the class of rings with nil-semicommutative contains rings with semicommutative. But the following example shows that the converse is not true.

Example 2.27. Clearly, Armendariz rings have nil-semicommutative. This is because, if $a b=0$ for some $a, b \in R$ and $r \in \operatorname{nil}(R)$ such that $r^{m}=0$ and $r^{k} \neq 0$ for $k<m$, then we have $(a-a r x)\left(b+r b x+r^{2} b x^{2}+\cdots+r^{m-1} b x^{m-1}\right)=0$. So arb $=0$ and hence $R$ has nil-semicommutative. In [18, Example 14], Huh, Lee, and Smoktunowicz found a ring which is Armendariz but does not have semicommutative.

One can find the next result in [16, Proposition 4.5(i)].

Proposition 2.28. Let $R$ be a ring, $(S, \leq)$ a strictly ordered monoid, and $\omega: S \rightarrow \operatorname{End}(R)$ a monoid homomorphism. Assume that $R$ is $(S, \omega)$-Armendariz and $\omega_{s}$ is compatible for some $s \in S \backslash\{1\}$. Then $R$ has nil-semicommutative.

Proposition 2.29. Let $R$ be a ring, $(S, \leq)$ a strictly ordered monoid, and $\omega: S \rightarrow E n d(R)$ a monoid homomorphism. If $R$ is $S$-compatible $(S, \omega)$-nil-Armendariz with nil $(R)$ nilpotent, then for all $f, g \in\left[\left[R^{S, \leq}, \omega\right]\right]$ and $h \in \operatorname{nil}\left(\left[\left[R^{S, \leq}, \omega\right]\right]\right)$, $f g=0$, implies $f h g=0$. 
Proof. Let $f, g \in\left[\left[R^{S, \leq}, \omega\right]\right]$ are such that $f g=0$ and $h^{k}=0$ for some positive integer $k$. We show that $f h g=0$. Since $h^{k}=0$ by Lemma 2.11, $\left(h\left(u_{1}\right)\right)^{k}=0$ for each $u_{1} \in S$. Since $f g=0$ and $R$ is $S$-compatible $(S, \omega)$-nilArmendariz, for every $u_{2}, u_{3} \in S$, we have $f\left(u_{2}\right) g\left(u_{3}\right)=0$. Hence by Proposition 2.28 and $h \in \operatorname{nil}\left(\left[\left[R^{S, \leq}, \omega\right]\right]\right)$ for each $u_{1}, u_{2}, u_{3}, s \in S$, we have

$$
(f h g)(s)=\sum_{\left(u_{2}, u_{1}, u_{3}\right) \in X_{s}(f, h, g)} f\left(u_{2}\right) h\left(u_{1}\right) g\left(u_{3}\right)=0 .
$$

Thus $f h g=0$.

Proposition 2.30. Let $R$ be a ring, $(S, \leq)$ a strictly ordered monoid, and $\omega: S \rightarrow E n d(R)$ a monoid homomorphism. Assume that $R$ is $S$-compatible $(S, \omega)$-nil-Armendariz with nil $(R)$ nilpotent. Then $R$ is nil-semicommutative if and only if $\left[\left[R^{S, \leq}, \omega\right]\right]$ is nil-semicommutative.

Proof. Suppose that $R$ is nil-semicommutative and $f, g \in\left[\left[R^{S, \leq}, \omega\right]\right]$ are such that $f g=0$. Since $R$ is $(S, \omega)$-nilArmendariz and nil-semicommutative, we have $f(u) R g(v)$

$=0$ for any $u \in \operatorname{supp}(f)$ and $v \in \operatorname{supp}(g)$. Now for any $h \in\left[\left[R^{S, \leq}, \omega\right]\right]$ and any $s \in S$,

$$
(f h g)(s)=\sum_{\left(u_{2}, u_{1}, u_{3}\right) \in X_{s}(f, h, g)} f\left(u_{2}\right) h\left(u_{1}\right) g\left(u_{3}\right)=0 .
$$

Thus $f h g=0$. This show that $f\left[\left[R^{S, \leq}, \omega\right]\right] g=0$. This means that $\left[\left[R^{S, \leq}, \omega\right]\right]$ is nil-semicommutative.

If $\left[\left[R^{S, \leq}, \omega\right]\right]$ is nil-semicommutative, then $R$ is nil-semicommutative since subrings of $n i l$-semicommutative rings are also nil-semicommutative.

Corollary 2.31. [45, Proposition 2.7] Let $(S, \leq)$ be a strictly ordered monoid and $R$ be an $S$-Armendariz ring. Then $R$ is semicommutative if and only if $\left[\left[R^{S, \leq}\right]\right]$ is semicommutative.

Proposition 2.32. Let $R$ be a ring, $(S, \leq)$ a strictly ordered monoid and $\omega: S \rightarrow E n d(R)$ a monoid homomorphism, and $I$ an ideal of $R$ (that is, $I \subseteq n i l(R)$ ). Assume that $R$ is a completely $S$-compatible ring. Then $R$ is $(S, \omega)$-nil-Armendariz if and only if $R / I$ is $(S, \bar{\omega})$-nil-Armendariz.

Proof. By analogy with the proof of [38, Proposition 5], we complete the proof.

Let $I$ be an index set and $R_{i}$ be a ring for each $i \in I$. Let $(S, \leq)$ be a strictly ordered monoid and $\omega^{i}: S \rightarrow$ $\operatorname{End}\left(R_{i}\right)$ a monoid homomorphism. Then the mapping $\omega: S \rightarrow \operatorname{End}\left(\prod_{i \in I} R_{i}\right)$ is a monoid homomorphism given by $\left.\omega_{s}\left(\left\{r_{i}\right\}_{i \in I}\right)=\left\{\left(\omega^{i}\right)_{s}\left(r_{i}\right)\right\}_{i \in I}\right\}$ for all $s \in S$.

Theorem 2.33. Let $R_{i}$ be a ring, $(S, \leq)$ a strictly ordered monoid, $\omega^{i}: S \rightarrow \operatorname{End}\left(R_{i}\right)$ a monoid homomorphism, for each $i$ in a finite index set $I$. If $R_{i}$ is $\left(S, \omega^{i}\right)$-nil-Armendariz for each $i$, then $R=\prod_{i \in I} R_{i}$ is $(S, \omega)$-nil-Armendariz, where $\omega=\prod_{i \in I} \omega^{i}$.

Proof. Let $R=\prod_{i \in I} R_{i}$ be the direct product of rings $\left(R_{i}\right)_{i \in I}$ and $R_{i}$ is $\left(S, \omega^{i}\right)$-nil-Armendariz for each $i \in I$. Denote the projection $R \rightarrow R_{i}$ as $\Pi_{i}$. Suppose that $f, g \in\left[\left[R^{S, \leq}, \omega\right]\right]$ are such that $f g \in\left[\left[\operatorname{nil}(R)^{S, \leq}, \omega\right]\right]$, where $\omega=\prod_{i \in I} \omega^{i}$. 
Set $f_{i}=\prod_{i} f, g_{i}=\prod_{i} g$. Then $f_{i}, g_{i} \in\left[\left[R_{i}^{S, \leq}, \omega^{i}\right]\right]$. For any $u, v \in S$, assume $f(u)=\left(a_{i}^{u}\right)_{i \in I}, g(v)=\left(b_{i}^{v}\right)_{i \in I}$. Now, for any $s \in S$,

$$
\begin{aligned}
(f g)(s) & =\sum_{(u, v) \in X_{s}(f, g)} f(u) \omega_{u}(g(v)) \\
& =\sum_{(u, v) \in X_{s}(f, g)}\left(a_{i}^{u}\right)_{i \in I}\left(\prod_{i \in I} \omega^{i}\right)_{u}\left(b_{i}^{v}\right)_{i \in I} \\
& =\sum_{(u, v) \in X_{s}(f, g)}\left(a_{i}^{u}\right)_{i \in I}\left(\prod_{i \in I} \omega_{u}^{i}\right)\left(b_{i}^{v}\right)_{i \in I} \\
& =\sum_{(u, v) \in X_{s}(f, g)}\left(a_{i}^{u} \omega_{u}^{i}\left(b_{i}^{v}\right)\right)_{i \in I} \\
& =\sum_{(u, v) \in X_{s}(f, g)}\left(f_{i}(u) \omega_{u}^{i}\left(g_{i}(v)\right)\right)_{i \in I} \\
& =\left(\sum_{(u, v) \in X_{s}(f, g)} f_{i}(u) \omega_{u}^{i}\left(g_{i}(v)\right)_{i \in I}\right. \\
& =\left(\sum_{(u, v) \in X_{s}\left(f_{i}, g_{i}\right)} f_{i}(u) \omega_{u}^{i}\left(g_{i}(v)\right)\right)_{i \in I} \\
& =\left(\left(f_{i} g_{i}\right)(s)\right)_{i \in I} .
\end{aligned}
$$

Since $(f g)(s) \in \operatorname{nil}(R)$, we have

$$
\left(f_{i} g_{i}\right)(s) \in \operatorname{nil}\left(R_{i}\right)
$$

Thus, $f_{i} g_{i} \in\left[\left[\operatorname{nil}\left(R_{i}\right)^{S, \leq}, \omega^{i}\right]\right]$. Now it follows $f_{i}(u) \omega_{u}^{i}\left(g_{i}(v)\right) \in \operatorname{nil}\left(R_{i}\right)$ for any $u, v \in S$ and any $i \in I$, since $R_{i}$ is $\left(S, \omega^{i}\right)$-nil-Armendariz. Hence, for any $u, v \in S$,

$$
f(u) \omega_{u}(g(v))=\left(f_{i}(u) \omega_{u}^{i}\left(g_{i}(v)\right)\right)_{i \in I} \in \operatorname{nil}(R)
$$

since $I$ is finite. This means that $R$ is $(S, \omega)$-nil-Armendariz.

Proposition 2.34. Let $R$ be a ring, $(S, \leq)$ a strictly ordered monoid and $\omega: S \rightarrow \operatorname{End}(R)$ a monoid homomorphism. Assume that $R$ is $S$-compatible and e an idempotent of $R$. If $e$ is central in $R$, then the following statements are equivalent:

(1) $R$ is $(S, \omega)$-nil-Armendariz;

(2) $e R$ and $(1-e) R$ are $(S, \omega)$-nil-Armendariz.

Proof. $(1) \Rightarrow(2)$. Is obvious since $e R$ and $(1-e) R$ are subrings of $R$.

$(2) \Rightarrow(1)$. Let $f, g \in\left[\left[R^{S, \leq}, \omega\right]\right]$ be such that $f g \in\left[\left[\operatorname{nil}(R)^{S, \leq}, \omega\right]\right]$. Let $f_{1}=C_{e} f, f_{2}=C_{(1-e)} f, g_{1}=C_{e} g, g_{2}=$ $C_{(1-e)} g$, where $f_{1}, f_{2} \in\left[\left[(e R)^{S, \leq}, \omega\right]\right]$ and $g_{1}, g_{2} \in\left[\left[((1-e) R)^{S, \leq}, \omega\right]\right]$. So $f_{1} g_{1}=C_{e} f C_{e} g=C_{e} f g \in \operatorname{nil}(e R)$ and $f_{2} g_{2}=C_{(1-e)} f C_{(1-e)} g=C_{(1-e)} f g \in \operatorname{nil}((1-e) R)$. Since $e R$ and $(1-e) R$ are $(S, \omega)$-nil-Armendariz, for each $u, v \in S$, implies that $f(u) \omega_{u}(g(v)) e \in \operatorname{nil}(e R)$ and $f(u) \omega_{u}(g(v))(1-e) \in \operatorname{nil}((1-e) R)$. Therefore $\left(f(u) \omega_{u}(g(v))\right)^{n} e$

$=\left(f(u) \omega_{u}(g(v))\right)^{m}(1-e)=0$, for some positive integers $n$ and $m$ and for each $u, v \in S$. So $\left(f(u) \omega_{u}(g(v))\right)^{k}=0$, for some positive max integer $k$ for $n$ and $m$. Thus, $f(u) \omega_{u}(g(v)) \in n i l(R)$. This means that $R$ is $(S, \omega)$-nilArmendariz.

\section{Weak annihilator of $(S, \omega)$-nil-Armendariz rings}

Let $R$ be a ring. For two subsets $U$ and $V$ of $R$. We use $U: V$ to represent the set $\{x \in R \mid V x \subseteq U\}$. Then for any $U \subseteq R$, we have

$$
\operatorname{nil}(R): U=\{x \in R \mid U x \subseteq \operatorname{nil}(R)\}
$$




$$
=\{x \in R \mid x U \subseteq \operatorname{nil}(R)\} .
$$

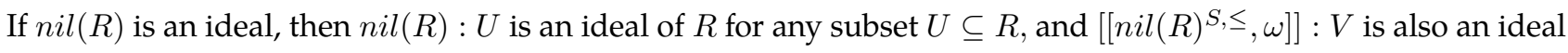
of $\left[\left[R^{S, \leq}, \omega\right]\right]$ for any subset $V$ of $\left[\left[R^{S, \leq}, \omega\right]\right]$. Given a skew generalized power series $f \in\left[\left[R^{S, \leq}, \omega\right]\right]$, let $C_{f}$ denote the set $\{f(s) \mid s \in \operatorname{supp}(f)\}$, and for a subset $V \subseteq\left[\left[R^{S, \leq}, \omega\right]\right]$, let $C_{V}$ denote the set $\cup_{f \in V} C_{f}$. Given a ring $R$, we define

$$
N A n n_{R}\left(2^{R}\right)=\{\operatorname{nil}(R): U \mid U \subseteq R\}
$$

and

$$
N A n n_{\left[\left[R^{S, \leq, \omega]]}\right.\right.}\left(2^{\left[\left[R^{S, \leq}, \omega\right]\right]}\right)=\left\{\left[\left[\operatorname{nil}(R)^{S, \leq}, \omega\right]\right]: V \mid V \subseteq\left[\left[R^{S, \leq}, \omega\right]\right]\right\}
$$

Proposition 3.1. Let $R$ be an $N I$ ring and $\operatorname{nil}(R)$ nilpotent, $(S, \leq)$ a strictly ordered monoid. Assume that $R$ is $S$ compatible and $(S, w)$-nil-Armendariz ring. Then

$$
\phi: N A n n_{R}\left(2^{R}\right) \rightarrow N A n n_{\left[\left[R^{S, \leq, \omega]]}\right.\right.}\left(2^{\left[\left[R^{S, \leq}, \omega\right]\right]}\right)
$$

defined by $\phi(I)=\left[\left[I^{S, \leq}, \omega\right]\right]$ for every $I \in N A n n_{R}\left(2^{R}\right)$ is bijective.

Proof. We first prove that $\left[\left[(\operatorname{nil}(R): U)^{S, \leq, \omega]}\right]=\left[\left[\operatorname{nil}(R)^{S, \leq, \omega]]}: U\right.\right.\right.$ for any subset $U \subseteq R$. Suppose that $f \in\left[\left[(\operatorname{nil}(R): U)^{S, \leq}, \omega\right]\right]$. Then for any $s \in S, f(s) \in \operatorname{nil}(R): U$, and so for any $u \in U$, by compatibility, $f(s) \omega_{s}(u)=\left(f C_{u}\right)(s) \in \operatorname{nil}(R)$. Hence $f C_{u} \in\left[\left[\operatorname{nil}(R)^{S, \leq}, \omega\right]\right]$, and so $f \in\left[\left[\operatorname{nil}(R)^{S, \leq, \omega]]}: U\right.\right.$. Thus $[[(\operatorname{nil}(R):$ $\left.\left.U)^{S, \leq}, \omega\right]\right] \subseteq\left[\left[\operatorname{nil}(R)^{S, \leq}, \omega\right]\right]: U$.

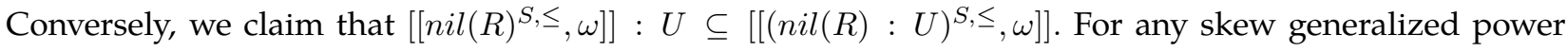
series $f \in\left[\left[\operatorname{nil}(R)^{S, \leq}, \omega\right]\right]: U$, and each $u \in U$, we have $f u=f C_{u} \in\left[\left[\operatorname{nil}(R)^{S, \leq}, \omega\right]\right]$. Then for any $s \in S$, and by compatibility, $\left(f C_{u}\right)(s)=f(s) \omega_{s}(u)=f(s) u \in \operatorname{nil}(R)$, and so for each $s \in S, f(s) \in \operatorname{nil}(R): U$. Hence $f \in\left[\left[(\operatorname{nil}(R): U)^{S, \leq}, \omega\right]\right]$. Thus, $\left[\left[\operatorname{nil}(R)^{S, \leq}, \omega\right]\right]: U \subseteq\left[\left[(\operatorname{nil}(R): U)^{S, \leq}, \omega\right]\right]$. Hence $\left[\left[\operatorname{nil}(R)^{S, \leq}, \omega\right]\right]: U=$ $\left[\left[(\operatorname{nil}(R): U)^{S, \leq}, \omega\right]\right]$ is proved, and so $\phi$ is well defined.

We next claim that $\phi$ is injective. Let

$$
\begin{aligned}
& I_{1}=\operatorname{nil}(R): U_{1} \in N A n n_{R}\left(2^{R}\right), \\
& I_{2}=\operatorname{nil}(R): U_{2} \in N A n n_{R}\left(2^{R}\right),
\end{aligned}
$$

and

$$
\operatorname{nil}(R): U_{1} \neq \operatorname{nil}(R): U_{2} .
$$

Then $\left[\left[\left(\operatorname{nil}(R): U_{1}\right)^{S, \leq}, \omega\right]\right] \neq\left[\left[\left(\operatorname{nil}(R): U_{2}\right)^{S, \leq}, \omega\right]\right]$ is clear. Hence $\phi\left(I_{1}\right) \neq \phi\left(I_{2}\right)$. So $\phi$ is injective.

Finally, we show that $\phi$ is surjective. Let

$$
\left[\left[\operatorname{nil}(R)^{S, \leq}, \omega\right]\right]: V \in N A n n_{\left[\left[R^{S, \leq, \omega]]}\right.\right.}\left(2^{\left[\left[R^{S,}, \omega\right]\right]}\right),
$$

where $V \in\left[\left[R^{S, \leq}, \omega\right]\right]$. We wish to claim that

$$
\left[\left[\operatorname{nil}(R)^{S, \leq}, \omega\right]\right]: V=\left[\left[\left(\operatorname{nil}(R): C_{V}\right)^{S, \leq}, \omega\right]\right]=\phi\left(\operatorname{nil}(R): C_{V}\right)
$$


Let $f \in\left[\left[\operatorname{nil}(R)^{S, \leq}, \omega\right]\right]: V$. Then $f g \in\left[\left[\operatorname{nil}(R)^{S, \leq}, \omega\right]\right]$ for any $g \in V$. Since $R$ is $(S, \omega)$-nil-Armendariz, $f(u) \omega_{u}(g(v)) \in \operatorname{nil}(R)$ for any $u, v \in S$. Thus by compatibility for any $u \in S, f(u) C_{V} \subseteq \operatorname{nil}(R)$ and so for any $u \in S, f(u) \in \operatorname{nil}(R): C_{V}$. Then $f \in\left[\left[\left(\operatorname{nil}(R): C_{V}\right)^{S, \leq}, \omega\right]\right]$ and so $\left[\left[\operatorname{nil}(R)^{S, \leq}, \omega\right]\right]: V \subseteq\left[\left[\left(\operatorname{nil}(R): C_{V}\right)^{S, \leq}, \omega\right]\right]$.

Conversely, assume that $f \in\left[\left[\left(\operatorname{nil}(R): C_{V}\right)^{S, \leq}, \omega\right]\right]$. Then for any $u \in S, f(u) C_{V} \subseteq \operatorname{nil}(R)$. Hence for any $g \in V$, it is easy to see that $f g \in\left[\left[\operatorname{nil}(R)^{S, \leq}, \omega\right]\right]$. So $f \in\left[\left[\operatorname{nil}(R)^{S, \leq}, \omega\right]\right]: V$. Hence $\left[\left[\left(\operatorname{nil}(R): C_{V}\right)^{S, \leq, \omega]} \subseteq \subseteq\right.\right.$ $\left[\left[\operatorname{nil}(R)^{S, \leq}, \omega\right]\right]: V$. Thus

$$
\left[\left[\operatorname{nil}(R)^{S, \leq}, \omega\right]\right]: V=\left[\left[\left(\operatorname{nil}(R): C_{V}\right)^{S, \leq}, \omega\right]\right]=\phi\left(\operatorname{nil}(R): C_{V}\right) .
$$

Hence $\phi$ is surjective. Therefore $\phi$ is a bijection.

A ring $R$ is called right zip provided that if the right annihilator $r_{R}(X)$ of a subset $X$ of $R$ is zero, then there exists a finite subset $Y \subseteq X$ such that $r_{R}(Y)=0$; equivalently, for a left ideal $J$ of $R$ with $r_{R}(J)=0$, there exists a finitely generated left ideal $J_{1} \subseteq J$ such that $r_{R}\left(J_{1}\right)=0 . R$ is zip if it is right and left zip. The concept of zip rings initiated by Zelmanowitz [21]. Zelmanowitz stated that any ring satisfying the descending chain condition on right annihilators is a right zip ring. Extensions of zip rings were studied by several authors. Faith in [6] proved that if $R$ is a commutative zip ring and $G$ is a finite abelian group, then the group $\operatorname{ring} R[G]$ of $G$ over $R$ is zip. Ced'o in [15] gave an example of a zip ring $R$ for which $R[x]$ is not zip. He also showed that there exists a right zip ring $R$ and finite group $\mathrm{G}$ such that the group ring $R[G]$ is not right zip. Faith in [6] raised the following questions:

(1) When does $R$ being a right zip ring imply $R[x]$ being right zip?

(2) When does $R$ being a right zip ring imply $R[G]$ being right zip when $G$ is a finite group?

Hong et al. in [7] proved that $R$ is a right zip ring if and only if $R[x]$ is a right zip ring when $R$ is an Armendariz ring. In [42], Cortes studied the relationship between right zip property of $R$ and skew polynomial extensions over $R$ by using the skew versions of Armendariz rings and generalized some results of Hong et al. In [28] O. Qun, introduced the notion of weak zip rings. A ring $R$ is a weak zip ring provided that for any subset $X$ of $R$, if $\operatorname{nil}(R): X \subseteq \operatorname{nil}(R)$, then there exists a finite subset $Y \subseteq X$ such that $\operatorname{nil}(R): Y \subseteq \operatorname{nil}(R)$. Isfahani [2] show that for a ring $R$, strictly ordered monoid $(S, \leq)$ and monoid homomorphism $\omega: S \rightarrow \operatorname{End}(R)$, if $R$ is $S$-compatible $(S, \omega)$-Armendariz, then $\left[\left[R^{S, \leq}, \omega\right]\right]$ is right zip if and only if $R$ is right zip. In the following we investigate the weak zip property of the skew generalized power series ring $\left[\left[R^{S, \leq}, \omega\right]\right]$ under the condition that $R$ is $(S, \omega)$-nil-Armendariz. The following we prove right weak zip and the left is similar.

Proposition 3.2. Let $R$ be an $N I$ ring and nil $(R)$ nilpotent, $(S, \leq)$ a strictly ordered monoid and $\omega: S \rightarrow$ End $(R) a$ monoid homomorphism. If $\left[\left[R^{S, \leq}, \omega\right]\right]$ is a right (left) weak zip ring and either of the following conditions holds:

(1) $\omega_{1}$ is compatible; or

(2) $\omega_{1}$ is automorphism.

Then $R$ is a right (left) weak zip ring.

Proof. Suppose that $A=\left[\left[R^{S, \leq}, \omega\right]\right]$ is a right weak zip ring and $\omega_{1}$ is compatible. Let $U \subseteq R$ with $r_{R}(U) \in \operatorname{nil}(R)$ and $U^{\prime}=\left\{c_{u} \mid u \in U\right\}$. If $f \in r_{A}\left(U^{\prime}\right)$, then $c_{u} f \in \operatorname{nil}(A)$ for each $u \in U$. Thus for each $s \in S$ and each $u \in U$, 
by Proposition 2.13, $c_{u}(1) \omega_{1}(f(s)) \in \operatorname{nil}(R)$. Since $\omega_{1}$ is compatible, $u f(s) \in \operatorname{nil}(R)$ for each $s \in \operatorname{supp}(f)$. Hence $f(s) \in r_{R}(U) \subseteq \operatorname{nil}(R)$ for each $s \in \operatorname{supp}(f)$. Then using Proposition 2.19, $f \in \operatorname{nil}(A)$. Therefore, $r_{A}\left(U^{\prime}\right) \subseteq \operatorname{nil}(A)$. Since $\left[\left[R^{S, \leq}, \omega\right]\right]$ is right weak zip, then it follows that there exists a finite set $V^{\prime} \subseteq U^{\prime}$ such that $r_{A}\left(V^{\prime}\right) \subseteq \operatorname{nil}(A)$, where $V^{\prime}=\left\{c_{r_{i}} \mid i=1,2, \ldots, n\right\}$ and $U^{\prime}=\left\{r_{i} \mid i=1,2, \ldots, n\right\}$. Now let $V=\left\{r \mid r \in U, c_{r} \in V^{\prime}\right\} . V$ is a finite subset of $U$. Now we show that $r_{R}(V) \in \operatorname{nil}(R)$. Let $r \in r_{R}(V)$. Since $\omega_{1}$ is compatible then $c_{r_{i}} \in r_{A}\left(V^{\prime}\right) \in \operatorname{nil}(A)$ for each $c_{r_{i}} \in V^{\prime}$ it follows that $\left(c_{r_{i}} f\right)(u)=r_{i} \omega_{0}(f(u))=r_{i} f(u) \in \operatorname{nil}(R)$ for each $u \in \operatorname{supp}(f)$ and $r_{i} \in U^{\prime} \subseteq U$. So, $T=\cup_{f \in r_{A}(V)}\{f(u) \mid u \in \operatorname{supp}(f) \subseteq \operatorname{nil}(R)\}$ and $R$ is a right weak zip ring.

Now assume that $A=\left[\left[R^{S, \leq}, \omega\right]\right]$ is a right weak zip ring and $\omega_{1}$ is automorphism. Let $U \subseteq R$ such that $r_{R}(U) \subseteq \operatorname{nil}(R)$ and let $U^{\prime}=\left\{c_{r} \in A \mid r \in U\right\}$. If $f \in r_{A}\left(U^{\prime}\right)$, then $c_{u} f \in \operatorname{nil}(A)$ for each $u \in U$. Thus, by Proposition 2.13 for each $s \in S$ and each $u \in U, \omega_{1}(u) \omega_{1}(f(s)) \in \operatorname{nil}(R)$. Since $\omega_{1}$ is automorphism, for each $s \in S, f(s) \in r_{R}(U)=0$ and so $r_{A}\left(U^{\prime}\right) \in \operatorname{nil}(A)$. Since $A$ is right weak zip, there exists a finite set $V^{\prime} \subseteq U^{\prime}$ such that $r_{A}\left(V^{\prime}\right) \in \operatorname{nil}(A)$. Now let $V=\left\{r \mid r \in U, c_{r} \in V^{\prime}\right\}$. $V$ is a finite subset of $U$. Now we show that $r_{R}(V) \in \operatorname{nil}(R)$. Let $r \in r_{R}(V)$ then for each $v \in V, v r=0$. Thus $c_{v} c_{r} \in \operatorname{nil}(A)$, for each $v \in V$ and so $c_{r} \in r_{A}\left(V^{\prime}\right) \in \operatorname{nil}(A)$. Hence $r_{R}(V) \in \operatorname{nil}(R)$. This means that $R$ is a right weak zip ring.

Theorem 3.3. Let $R$ be a ring, $(S, \leq)$ a strictly ordered monoid and $\omega: S \rightarrow \operatorname{End}(R)$ a monoid homomorphism. If $R$ is a right (left) weak zip $S$-compatible and $(S, \omega)$-nil-Armendariz with nil $(R)$ nilpotent, then $\left[\left[R^{S, \leq}, \omega\right]\right]$ is a right (left) weak zip ring.

Proof. Suppose that $R$ is a right weak zip ring and put $A=\left[\left[R^{S, \leq}, \omega\right]\right]$. Let $U \subseteq\left[\left[R^{S, \leq}, \omega\right]\right]$ such that $r_{A}(U) \subseteq$ $\operatorname{nil}(A)$. We first show that $\operatorname{nil}(U) \subseteq \operatorname{nil}(R)$. Now let $V=\{f(s) \mid f \in U, s \in S\}$. If $r \in r_{R}(V)$, then $f(s) r \in \operatorname{nil}(R)$ for any $f \in U$ and $s \in S$. Since $\operatorname{nil}(R)$ nilpotent and $R$ is $S$-compatible, $f(s) \omega_{s}(r)=\left(f c_{r}\right)(s) \in \operatorname{nil}(R)$ for any $s \in S$. Then by using Proposition 2.19, $f c_{r} \in \operatorname{nil}(A)$. Hence $c_{r} \in r_{A}(U) \subseteq \operatorname{nil}(A)$. Therefore by Proposition 2.13, $r \in \operatorname{nil}(R)$. Thus, $\operatorname{nil}(U) \subseteq \operatorname{nil}(R)$. Since $R$ is right weak zip, there exists a finite set $V^{\prime} \subseteq V$ such that $r_{R}\left(V^{\prime}\right) \subseteq \operatorname{nil}(R)$. Without loss of generality, we may assume that $V^{\prime}=\left\{v_{1}, v_{2}, \ldots, v_{n}\right\} \subseteq C_{V}$. For each $v_{i} \in V^{\prime}$, there exists some $g_{v_{i}} \in U$ such that $v_{i}=g_{v_{i}}\left(s_{i}\right)$, for some $s_{i} \in S$. Let $U^{\prime}$ be a minimal subset of $U$ such that $g_{v_{i}} \in U^{\prime}$ for each $v_{i} \in V^{\prime}, 1 \leq i \leq n$. Then $U^{\prime}$ is a nonempty finite subset of $U$ and $V^{\prime} \subseteq V$. Now we show that $\operatorname{nil}\left(U^{\prime}\right) \subseteq \operatorname{nil}(A)$. Let $V^{\prime \prime}=\left\{f(s) \mid f \in U^{\prime}, s \in S\right\}$. Then $V^{\prime} \subseteq V^{\prime \prime}$ and so $r_{R}\left(V^{\prime \prime}\right) \subseteq r_{R}\left(V^{\prime}\right) \subseteq \operatorname{nil}(R)$. Suppose that $f \in \operatorname{nil}\left(U^{\prime}\right)$, then $f g \in \operatorname{nil}(A)$ for each $f \in U^{\prime}$. Using Proposition 2.13, $(f g)(s) \in \operatorname{nil}(R)$ for each $s \in \operatorname{supp}(f g)$. Tracing the same procedure used in Proposition 3.1, we can show that $g(v) f(u) \in \operatorname{nil}(R)$ for each $u, v \in S$ since $R$ is $(S, \omega)$-nil-Armendariz and $S$-compatible, and so for any $u \in S, f(u) \in \operatorname{nil}(R)$. Hence by Proposition 2.19, $f \in \operatorname{nil}(A)$. Therefore $\operatorname{nil}\left(U^{\prime}\right) \subseteq \operatorname{nil}(A)$, as desired.

Theorem 3.4. Let $R$ be a ring, $(S, \leq)$ a strictly ordered monoid and $\omega: S \rightarrow \operatorname{End}(R)$ a monoid homomorphism. If $R$ is a right (left) weak zip and $(S, \omega)$-nil-Armendariz with nil $(R)$ nilpotent and $\omega_{s}$ is automorphism for each $s \in S$, then $\left[\left[R^{S, \leq}, \omega\right]\right]$ is a right (left) weak zip ring.

Proof. Suppose that $R$ is a right weak zip ring and put $A=\left[\left[R^{S, \leq}, \omega\right]\right]$. Let $U \subseteq\left[\left[R^{S, \leq}, \omega\right]\right]$ with $r_{A}(U) \subseteq \operatorname{nil}(A)$. Now let $V=\left\{\omega_{s}^{-1}(f(s)) \mid f \in U, s \in S\right\}$. If $r \in r_{R}(V)$, then $\omega_{s}^{-1}(f(s)) r=0$ for each $f \in U$ and $s \in S$. Thus, for each $f \in U$ and $s \in S, f(s) \omega_{s}(r) \in \operatorname{nil}(R)$ and so $\left(f c_{r}\right)(s) \in \operatorname{nil}(R)$ since $\operatorname{nil}(R)$ nilpotent. Then 
$c_{r} \in r_{A}(U) \subseteq \operatorname{nil}(A)$ and hence $r_{R}(V) \subseteq \operatorname{nil}(R)$. Since $R$ is right weak zip, there exists a finite set $V_{0} \subseteq V$ such that $r_{R}\left(V_{0}\right)=0$. For each $v \in V_{0}$, there exists $g_{v} \in U$ such that $v=\omega_{s}^{-1}\left(g_{v}(s)\right)$, for some $s \in S$. Let $U_{0}$ be a minimal subset of $U$ such that $g_{v} \in U_{0}$ for each $v \in V_{0}$. Then $U_{0}$ is a nonempty finite subset of $U$. Let $V_{1}=\left\{\omega_{s}^{-1}(f(s)) \mid f \in U_{0}, s \in S\right\}$. Then $V_{0} \subseteq V_{1}$ and so $r_{R}\left(V_{1}\right) \subseteq r_{R}\left(V_{0}\right) \subseteq \operatorname{nil}(R)$. If $f \in r_{A}\left(U_{0}\right) \subseteq \operatorname{nil}(A)$ then $g f \in \operatorname{nil}(A)$ for any $g \in U_{0}$. Since $R$ is $(S, \omega)$-nil-Armendariz, then $g(s) \omega_{s}(f(t)) \in \operatorname{nil}(R)$ for each $s, t \in S$. Thus $\omega_{s}^{-1}(g(s)) f(t) \in \operatorname{nil}(R)$ for each $s, t \in S$ and so for each $t \in S, f(t) \in r_{R}\left(V_{1}\right) \subseteq \operatorname{nil}(R)$. Then $r_{A}\left(U_{0}\right) \subseteq \operatorname{nil}(A)$. This means that $\left[\left[R^{S, \leq}, \omega\right]\right]$ is a right weak zip ring.

Proposition 3.5. Let $R$ be a right Noetherian NI ring, $(S, \leq)$ a strictly ordered monoid and $\omega: S \rightarrow$ End $(R)$ a monoid homomorphism. Assume that $R$ is $S$-compatible $(S, \omega)$-nil-Armendariz with nil $(R)$ nilpotent, then the following conditions are equivalent:

(1) $R$ is a weak zip ring.

(2) $\left[\left[R^{S, \leq}, \omega\right]\right]$ is a weak zip ring.

Proof. The proof follows from Proposition 2.13, 2.19 and 3.1.

Corollary 3.6. [29, Proposition 3.12] Let $(S, \leq)$ be a cancellative torsion-free strictly ordered monoid and $R$ a nil generalized power serieswise Armendariz ring with nil $(R)$ nilpotent. Then the following conditions are equivalent:

(1) $R$ is a weak zip ring.

(2) $\left[\left[R^{S, \leq}\right]\right]$ is a weak zip ring.

Corollary 3.7. [29, Corollary 3.13] We have the following results.

(1) If $R$ is a nil power serieswise Armendariz with nil $(R)$ nilpotent. Then $R$ is a weak zip ring if and only if the power series ring $R[[x]]$ is weak zip.

(2) If $R$ is an $n$ nil power serieswise Armendariz with nil $(R)$ nilpotent. Then $R$ is a weak zip ring if and only if the power series ring $R\left[\left[x_{1}, \ldots, x_{n}\right]\right]$ in $n$ indeterminates is weak zip.

Cohn in [31] calls reversible rings, those rings $R$ with the property that for all $a, b \in R, a b=0$ implies $b a=0$. A stronger condition than "reversible" was defined by Lambaek in [21]. A ring $R$ is called symmetric if, for all $a, b, c \in R, a b c=0$ implies $a c b=0$. Cohn in [31] shows that the Kothe's conjecture is true for the class of reversible rings. In the following, we investigate the weak symmetric property of the rings of skew generalized power series.

Definition 3.8. [30] A ring $R$ is called a weak symmetric ring if abc $\in \operatorname{nil}(R), a c b \in \operatorname{nil}(R)$ for all $a, b, c \in R$.

Definition 3.9. [43] A ring $R$ is called weakly reversible, if for all $a, b, r \in R$ such that $a b=0$, Rbra is nil left ideal of $R$ (equivalently, braR is nil right of $R$ ).

Theorem 3.10. Let $R$ be an $N I$ ring with nil $(R)$ nilpotent, $(S, \leq)$ a strictly ordered monoid, and $\omega: S \rightarrow$ End $(R) a$ monoid homomorphism. Assume that $R$ is $(S, \omega)$-nil-Armendariz and $S$-compatible. Then $R$ is a weak symmetric if and only if $\left[\left[R^{S, \leq}, \omega\right]\right]$ is a weak symmetric. 
Proof. Since any subring of a weak symmetric ring is again a weak symmetric ring, it suffices to show that if $R$ is a weak symmetric ring, then so is $\left[\left[R^{S, \leq}, \omega\right]\right]$. Let $f, g, h \in\left[\left[R^{S, \leq}, \omega\right]\right]$ be such that $f g h \in \operatorname{nil}\left(\left[\left[R^{S, \leq}, \omega\right]\right]\right)$. By Proposition 2.13, we have $f(u) g(v) h(t) \in \operatorname{nil}(R)$ for all $u, v, t \in S$, and so $f(u) h(t) g(v) \in \operatorname{nil}(R)$ for all $u, t, v \in S$ since $R$ is weak symmetric. Hence $f h g \in \operatorname{nil}\left(\left[\left[R^{S, \leq}, \omega\right]\right]\right)$ by Proposition 2.19. Therefore $\left[\left[R^{S, \leq}, \omega\right]\right]$ is a weak symmetric ring.

Proposition 3.11. Let $R$ be an $N I$ ring with nil $(R)$ nilpotent, $(S, \leq)$ a strictly ordered monoid, and $\omega: S \rightarrow$ End $(R) a$ monoid homomorphism. Assume that $R$ is $(S, \omega)$-nil-Armendariz and $S$-compatible. Then $R$ is weakly reversible if and only if $\left[\left[R^{S, \leq}, \omega\right]\right]$ is weakly reversible.

Proof. The proof is similar to that of Proposition 3.10.

The following corollary will give more examples of weak zip rings, weak symmetric rings and weakly reversible rings.

Corollary 3.12. Let $R$ be an $N I$ ring and nil $(R)$ nilpotent, $\left(S_{1}, \leq_{1}\right),\left(S_{2}, \leq_{2}\right), \cdots,\left(S_{n}\right.$,

$\left.\leq_{n}\right)$ be a strictly ordered monoids, and $\omega^{i}: S_{i} \rightarrow \operatorname{End}(R)$ a monoid homomorphisms. Assume that $R$ is $S_{i}$-compatible $(i=$ $1, \ldots, n)$ and $(S, \omega)$-nil-Armendariz. Denote by $($ lex $\leq)$ and(revlex $\leq)$ the lexicographic order, the reverse lexicographic order, respectively, on the monoid $S_{1} \times S_{2} \times \cdots \times S_{n}$. Then we have the following:

(1) $R$ is a weak zip $\Leftrightarrow\left[\left[R^{S_{1} \times S_{2} \times \cdots \times S_{n},(l e x \leq)}, \omega\right]\right]$ is a weak zip.

(2) $R$ is a weak zip $\Leftrightarrow\left[\left[R^{S_{1} \times S_{2} \times \cdots \times S_{n},(\text { revlex } \leq)}, \omega\right]\right]$ is a weak zip.

(3) $R$ is a weak symmetric $\Leftrightarrow\left[\left[R^{S_{1} \times S_{2} \times \cdots \times S_{n},(l e x \leq)}, \omega\right]\right]$ is a weak symmetric.

(4) $R$ is a weak symmetric $\Leftrightarrow\left[\left[R^{S_{1} \times S_{2} \times \cdots \times S_{n},(\text { revlex } \leq)}, \omega\right]\right]$ is a weak symmetric.

(5) $R$ is a weakly reversible $\Leftrightarrow\left[\left[R^{S_{1} \times S_{2} \times \cdots \times S_{n},(l e x \leq)}, \omega\right]\right]$ is a weakly reversible.

(6) $R$ is a weakly reversible $\Leftrightarrow\left[\left[R^{S_{1} \times S_{2} \times \cdots \times S_{n},(\text { revlex } \leq)}, \omega\right]\right]$ is a weakly reversible.

Proof. It is easy to see that $\left(S_{1} \times S_{2} \times \cdots \times S_{n},(\right.$ lex $\left.\leq)\right)$ and $\left(S_{1} \times S_{2} \times \cdots \times S_{n},(\right.$ revlex $\left.\leq)\right)$ are strictly ordered monoids. Therefore we complete the proofs of (1), (2) by Proposition 3.5, and (3),(4), (5), (6) by Proposition 3.10.

\section{REFERENCES}

[1] A. Smoktunowicz, Polynomial rings over nil rings need not be nil. J. Algebra, 233 (2000), 427-436.

[2] A. R. Nasr-Isfahani, Radical of skew generalized power series rings, J. Algebra. Appl, 12(1) (2013), 1250129(1-13).

[3] A. Alhevaz and A. Moussavi, On Monoid Rings Over Nil Armendariz Ring, Comm Algebra, 42(1)(2013), 1-21.

[4] Amitsur, A, Algebras over infinite fields. Proc. Amer. Math. Soc, 7 (1956), 35-48.

[5] Bell, H. E, Near-rings in which each element is a power of itself. Bull. Aust. Math. Soc, 2 (1970), 363-368.

[6] C. Faith, Rings with zero intersection property on annihilators: Zip rings, Publ. Mat, 33 (1989), 329-332.

[7] C. Y. Hong, N. K. Kim, T. K. Kwak and Y. Lee, Extensions of zip rings, J. Pure Appl. Algebra, 195 (2005), 231-242.

[8] C. Huh, Y. Lee and A. Smoktunowicz, Armendariz rings and semicommutative rings, Comm. Algebra, 30(2) (2002), 751-761.

[9] C. Y. Hong, N. K. Kim, and T. K. Kwak, 'On skew Armendariz rings', Comm. Algebra, 31(1) (2003), 103-122.

[10] C. Y. Hong, N. K. Kim, and T. K. Kwak, Ore extensions of Baer and p.p.-rings, J. Pure Appl. Algebra, 151 (2000), $215-226$.

[11] D. D. Anderson and V. Camillo, Armendariz rings and Gaussian rings, Comm. Algebra, 26 (7) (1998), $2265-2272$.

[12] E. Hashemi, A. Moussavi, Polynomial extensions of quasi-Baer rings, Acta Math. Hungar,107(3) (2005), 207-224. 
[13] E. P. Armendariz, A note on extensions of Baer and p.p.-rings, J. Austral. Math. Soc, 18 (1974), 470-473.

[14] E. Ali and Z. K. Liu, On the ring theory of skew generalized power series, (P.hD)(June, 2015), North west Normal Uni, China.

[15] F. Ced'o, Zip rings and MalŠcev domains, Commun. Algebra, 19 (1991), 1983-1991.

[16] G. Marks, R. Mazurik, M. Ziembowski, A unified approach to various generalizations of Armendariz rings., Bull. Aust. Math. Soc. 81 (2010), 361-397.

[17] G. Marks, R. Mazurek and M. Ziembowski, 'A new class of unique product monoids with applications to ring theory', Semigroup Forum 78(2) (2009), 210-225.

[18] Huh, C., Lee, Y., Smoktunowicz, A, Armendariz rings and semicommutative rings. Comm Algebra,30 (2002), 751-761.

[19] J. Esmaeili, E. Hashemi, On Nilpotent Elements of Skew Polynomial Rings, J. Math Ext, 6 (3) (2012), 1-15.

[20] J. M. Zelmanowitz, The finite intersection property on annihilator right ideals, Proc. Amer. Math. Soc, 57 (1976), $213-216$.

[21] J. Krempa, Some examples of reduced rings, Algebra Colloq.,3 (1996), 289-300.

[22] K. Paykan, A. Moussavi, M. ZahiriZ, Special properties of rings of skew generalized power series, Comm. Algebra, 42(12) (2014), 5224-5248.

[23] K. Paykan, A. Moussavi, Nilpotent elements and nil-Armendariz property of skew generalized power series rings, Asian-European Journal of Mathematics, 10(1) (2017) 1750034.

[24] M. Ferrero, R. Mazurek, A. SantŠAna, On right chain semigroups, J. Algebra, 292 (2005), 574-584.

[25] M. B. Rege and S. Chhawchharia, Armendariz rings, Proc. Japan Acad. Ser. A math. Sci, 73 (1997), 14-17.

[26] N. K. Kim and Y. Lee, Armendariz rings and reduced rings, J. Algebra, 223 (2000), 477-488.

[27] N. K. Kim, K. H. Lee, and Y. Lee, Power series rings satisfying a zero divisor property, Comm. Algebra, 34(6) (2006), $2205-2218$.

[28] O. L. Qun, Ore extensions of weak zip rings, Glasg. Math. J, 51 (3) (2009), 525-537.

[29] O. L. Qun, L. Wang, On nil generalized power serieswise Armendariz rings, Comm. Korean Math. Soc, 28 (3) (2013), $463-480$.

[30] O. L. Qun, C. Huanyin, On Weak Symmetric Rings, Comm. Algebra, 38(2) (2010), 697-713.

[31] P. M. Cohn, Reversible rings, Bull. London Math. Soc, 31 (1999), 641-648.

[32] Ribenboim. P, Noetherian rings of generalized power series. J. Pure Appl. Algebra, 79 (1992), 293-312.

[33] Ribenboim. P, Rings of generalized power series: Nilpotent elements. Abh. Math. Sem. Univ. Hamburg, 61 (1991), 15-33.

[34] R. Mazurek and M. Ziembowski, Uniserial rings of skew generalized power series, J. Algebra, 318 (2) (2007), 737-764.

[35] R. Mazurek and M. Ziembowski, 'On von Neumann regular rings of skew generalized power series', Comm. Algebra, 36 (5) (2008), 1855-1868.

[36] R. Antoine, Nilpotent elements and Armendariz rings, J. Algebra, 319 (2008), 3128-3140.

[37] R.M. Salem, Prüer rings of generalized power series, Southeast Asian Bull. Math,33 (2009), 527-534.

[38] S. Hizem, A note on nil power serieswise Armendariz rings, Rendiconti del Circolo Mathematico di Palermo, 59 (1) (2010), 87-99.

[39] S. A. Annin, Attached primes under skew polynomial extension, J. Algebra Appl,10 (3) (2011), 537-547.

[40] Shin, G, Prime ideal and sheaf representation of a pseudo symmetric rings. Trans. Amer. Math. Soc, 184 (1973), 43-60.

[41] T. K. Lee and T. L, Wong, On Armendariz rings, Houston J. Math, 29 (2003), 583-593.

[42] W. Cortez, Skew polynomial extensions over zip rings, Int. J. Math. Math. Sci, 9 (2008), Article ID: 466720.

[43] Z. Liang and G. Yang, On weakly reversible ring, Acta Math. Univ. Comenianae, 76 (2) (2007), 189-192.

[44] Z. K. Liu and R. Zhao, On weak Armendariz rings, Comm. Algebra, 34 (7) (2006), 2607-2616.

[45] Z. K. Liu, Special properties of rings of generalized power series, Comm. Algebra, 32 (8) (2004), 3215-3226. 